\title{
Guest Editorial: Special Issue on Latin American Theoretical Informatics Symposium (LATIN)
}

\author{
Eduardo Sany Laber • Claudson Bornstein • \\ Cristina Fernandes
}

Received: 27 October 2010 / Accepted: 1 November 2010 / Published online: 20 November 2010 (C) Springer Science+Business Media, LLC 2010

This special issue of Algorithmica contains expanded journal articles of selected papers from the eighth Latin American Theoretical INformatics conference (LATIN), which was held in the charming city of Buzios, Rio de Janeiro, Brazil, in April 2008.

LATIN was launched in 1992 to foster the interaction between the Latin-American community and computer scientists around the world. The conference is now held every other year, and LATIN'08 was the eighth of a series, after São Paulo, Brazil (1992); Valparaiso, Chile (1995); Campinas, Brazil (1998); Punta del Este, Uruguay (2000); Cancún, Mexico (2002); Buenos Aires, Argentina (2004); and Valdivia, Chile (2006). In the past two decades, LATIN has established itself as a theoretical computer science conference of the highest standards, and this special issue reflects this fact.

LATIN'08 had more than 120 participants among students and researchers from academia and industry. Its conference proceedings were published, as with all past LATINs, by Springer-Verlag in the Lecture Notes in Computer Science series. There were sixty six papers presented in LATIN'08, and Volume 4957 of the LNCS series is devoted to them.

This special issue includes the following expanded papers:

- Origami Embedding of Piecewise-Linear Two-Manifolds. Marshall Bern and Barry Hayes

- On Dissemination Thresholds in Regular and Irregular Graph Classes. Ivan Rapaport, Karol Suchan, Ioan Todinca and Jacques Verstraete

- Quantum Property Testing of Group Solvability. Yoshifumi Inui and François Le Gall

E.S. Laber $(\bowtie) \cdot$ C. Bornstein · C. Fernandes

PUC-Rio, Rio de Janeiro, Brazil

e-mail: laber@inf.puc-rio.br 
- Random 2 XORSAT Phase Transition. Hervé Daudé and Vlady Ravelomanana

- Making Doubling Metrics Geodesic. Anupam Gupta and Kunal Talwar

- Approximation Algorithms for k-Hurdle Problems. Brian C. Dean, Adam Griffis and Adam Whitley

- Energy Efficient Monitoring in Sensor Networks. Amol Deshpande, Samir Khuller, Azarakhsh Malekian and Mohammed Toossi

These selected papers are amongst the best presented in LATIN'08, and provide a wide view of the different subfields of TCS in the scope of LATIN. Each of the above papers is an extended and thoroughly revised version of the conference paper, which went through a careful refereeing process. We are grateful to the authors for their valuable contributions, as well as the anonymous reviewers for their effort and dedication.

We look forward to the many successfull LATIN conferences that are yet to come. 